CITA-2002-09, hep-th/0204187

\title{
Prospects and Problems of Tachyon Matter Cosmology
}

\author{
Andrei Frolov \\ Canadian Institute for Theoretical Astrophysics, University of Toronto \\ Toronto, ON, M5S 3H8, Canada \\ Lev Kofman \\ Canadian Institute for Theoretical Astrophysics, University of Toronto \\ Toronto, ON, M5S 3H8, Canada \\ Alexei Starobinsky \\ Landau Institute for Theoretical Physics, Kosygina 2, \\ Moscow, 117334, Russia
}

(April 22, 2002)

\begin{abstract}
We consider the evolution of FRW cosmological models and linear perturbations of tachyon matter rolling towards a minimum of its potential. The tachyon coupled to gravity is described by an effective $4 \mathrm{~d}$ field theory of string theory tachyon. In the model where a tachyon potential $V(T)$ has a quadratic minimum at finite value of the tachyon field $T_{0}$ and $V\left(T_{0}\right)=0$, the tachyon condensate oscillates around its minimum with a decreasing amplitude. It is shown that its effective equation of state is $p=-\varepsilon / 3$. However, linear inhomogeneous tachyon fluctuations coupled to the oscillating background condensate are exponentially unstable due to the effect of parametric resonance. In another interesting model, where tachyon potential exponentially approaches zero at infinity of $T$, rolling tachyon condensate in an expanding universe behaves as pressureless fluid. Its linear fluctuations coupled with small metric perturbations evolve similar to these in the pressureless fluid. However, this linear stage changes to a strongly non-linear one very early, so that the usual quasi-linear stage observed at sufficiently large scales in the present Universe may not be realized in the absence of the usual particle-like cold dark matter.
\end{abstract}

Typeset using REVTEX 


\section{INTRODUCTION}

There are many faces of superstring/brane cosmology which come from different corners of M/String theories. In particular, people search for potential candidates to explain early universe inflation, present day dark energy and dark matter in the universe. One of the string theory constructions, tachyon on D-branes, has been recently proposed for cosmological applications by Sen [四]. A relatively simple formulation of the unstable D-brane tachyon dynamics in terms of effective field theory stimulates one to investigate its role in cosmology [2].

The rolling tachyon in the string theory may be described in terms of effective field theory for the tachyon condensate $T$, which in the flat spacetime has a Lagrangian density

$$
\mathcal{L}=-V(T) \sqrt{1+\partial_{\mu} T \partial^{\mu} T}
$$

The tachyon potential $V(T)$ has a positive maximum at $T=0$ and a minimum at $T_{0}$, with $V\left(T_{0}\right)=0$. We consider two models, with finite value of $T_{0}$ and with minimum at infinity, as illustrated in Figure 1 . In both cases one encounters interesting possibilities for cosmological applications.

In the case of finite $T_{0}$, we consider quadratic expansion around the minimum of the potential $V(T) \approx \frac{1}{2} m^{2}\left(T-T_{0}\right)^{2}$. As we will show, in this case the tachyon matter has negative pressure and may be considered a candidate for quintessence.

In the case when $T_{0} \rightarrow \infty$, we use exponential asymptotic of the potential $V(T)=$ $V_{0} e^{-T / T_{0}}$ derived from the string theory calculations [3,4] (exact form of the potential from Ref. [3] is $V=\left(1+\frac{T}{T_{0}}\right) e^{-T / T_{0}}$; our qualitative results for late time asymptotics of $T(t)$ do not depend on the pre-exponential factor). Dimensional parameters of the potential are related to the fundamental length scale, $T_{0} \sim l_{s}$, and $V_{0}$ is the brane tension. As it was demonstrated by Sen [4], the tachyon matter is pressureless for the potential with the ground state at infinity. In this case tachyon matter may be considered a cold dark matter candidate [1].

It is noteworthy that the models of type (1) already were studied in cosmology on the phenomenological ground. For certain choices of potentials $V$ and non-minimal kinetic terms one can get kinematically driven inflation, " $k$-inflation" [5]. In particular, a toy model with the potential $V(T) \sim 1 / T^{2}$ with ground state at infinity may give rise to the power law inflation of the universe [5]. However, it remains to be seen how this potential can be motivated by the string theory of tachyon. The model with $V \equiv$ const is reduced to the so-called "Chaplygin gas" where the matter equation of state is $p=-$ const $/ \varepsilon$. Such matter was suggested as a candidate for the present dark energy [8,9].

In this paper, we investigate cosmology with tachyon matter with the string theory motivated potentials of Figure 11. In Section [1], we write down the equations for the tachyon matter coupled to gravity. We focus on self-consistent formulation of the isotropic FriedmannRobertson-Walker cosmology supported by the tachyon matter. It is described by coupled equations for the time-dependent background tachyon field $T(t)$ and the scale factor of the universe $a(t)$.

One of the lessons of the scalar field theory in cosmology is the possibility of the fast growth of inhomogeneous scalar field fluctuations, as it was found in different situations. 


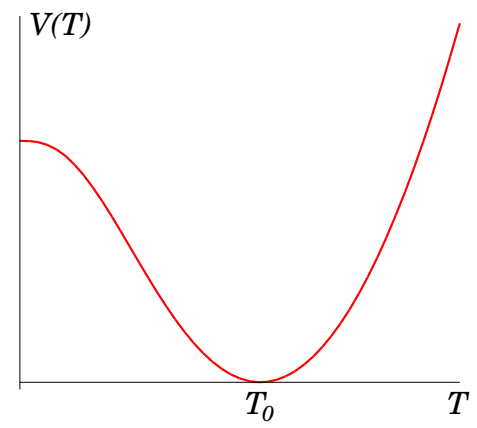

(a)

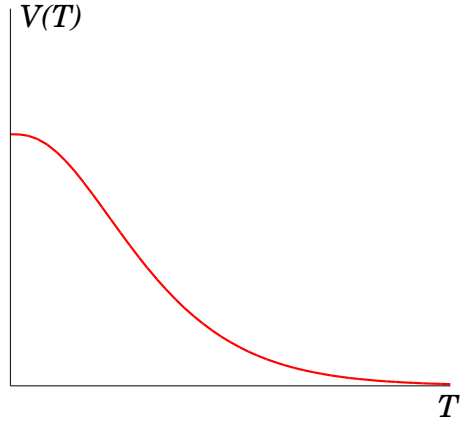

(b)

FIG. 1. Tachyon matter potentials with minima at finite (a) and infinite (b) values of the field. The potentials near minimum are taken to be: (a) $V(T)=\frac{1}{2} m^{2}\left(T-T_{0}\right)^{2}$, (b) $V(T)=V_{0} e^{-T / T_{0}}$.

Instability of scalar field fluctuations are typical for preheating after inflation due to the parametric resonance [10], or tachyonic preheating after hybrid inflation [11] (which so far has only remote relation with string theory tachyon). Fluctuations can be unstable in axion cosmology due to the parametric resonance [12]. Therefore, we address the problem of stability of linear fluctuations of the rolling tachyon matter.

Consistent investigation of tachyon cosmology, in principle, should be started with the tachyon rolling from the top of its potential, which has negative curvature. In this case the setting of the problem is similar to what we met in tachyonic preheating after hybrid inflation [11]. In these cases we expect fast decay of the scalar field into long-wavelength inhomogeneities. Here we assume that somehow homogeneous tachyon rolls towards the minimum of its potential as universe expands, and consider tachyon fluctuations at the latest stages of its evolution.

In Section IIT, we develop a formalism for treating small fluctuations of the tachyon field $\delta T(t, \vec{x})$. It is possible to extend theory of tachyon matter fluctuations by including scalar metric fluctuations. This allows us to address the issue of gravitational instability in tachyon cosmology. Applying this analysis for specific tachyon potentials, we will see that instability of tachyon fluctuations is essential for the whole story of tachyon cosmology.

In Section IV, we consider background cosmological solutions for the tachyon potential with the ground state at finite value $T_{0}$. We find that tachyon field is oscillating around the minimum of its potential, while its equation of state (averaged over oscillations) is $p=-\frac{1}{3} \varepsilon$. Then in Section V, we check the stability of tachyon fluctuations around this background solutions, and find that they are exponentially unstable due to the parametric resonance.

In Section V1, we repeat the analysis for the model where tachyon potential is exponential $V(T) \propto e^{-T / T_{0}}$ and its ground state is at $T \rightarrow \infty$. In this case, background cosmological solution corresponds to the pressureless tachyon with energy density $\varepsilon \propto 1 / a^{3}$. In Section VII, we consider small inhomogeneous tachyon and metric fluctuations, and find gravitational instability of fluctuations around the background solution. Specifically, we find that for the pressureless rolling tachyon linear approximation for fluctuations becomes insufficient very early. We argue that the rolling tachyon dark matter scenario may have difficulties in explaining gravitational clustering and the large scale velocity flows. 


\section{COSMOLOGY WITH ROLLING TACHYON MATTER}

Rolling tachyon is associated with unstable D-branes, and self-consistent inclusion of gravity may require higher-dimensional Einstein equations with branes. Still, in the low energy limit, one expects that the brane gravity is reduced to the four dimensional Einstein theory [13].

In this section, we consider tachyon matter coupled with Einstein gravity in four dimensions. Tachyon matter is described by the phenomenological Lagrangian density (1), where derivatives are covariantly generalized with respect to the metric $g_{\mu \nu}, \partial_{\mu} \rightarrow \nabla_{\mu}$. We use the metric with signature $(-,+,+,+)$. The model is given by the action

$$
S=\int d^{4} x \sqrt{-g}\left(\frac{R}{16 \pi G}-V(T) \sqrt{1+\nabla_{\mu} T \nabla^{\mu} T}\right) .
$$

The Einstein equations which follow from (2) are

$$
R_{\mu \nu}-\frac{1}{2} g_{\mu \nu} R=8 \pi G\left(\frac{V}{\sqrt{1+\nabla_{\alpha} T \nabla^{\alpha} T}} \nabla_{\mu} T \nabla_{\nu} T-g_{\mu \nu} V \sqrt{1+\nabla_{\alpha} T \nabla^{\alpha} T}\right),
$$

and the field equation for the tachyon is

$$
\nabla_{\mu} \nabla^{\mu} T-\frac{\nabla_{\mu} \nabla_{\nu} T}{1+\nabla_{\alpha} T \nabla^{\alpha} T} \nabla^{\mu} T \nabla^{\nu} T-\frac{V_{, T}}{V}=0 .
$$

Let us apply these equations for the isotropic and homogeneous FRW cosmological model

$$
d s^{2}=-d t^{2}+a^{2}(t) d \vec{x}^{2}
$$

where $a(t)$ is the scale factor of the spatially flat $(K=0)$ universe. For this geometry, the energy-momentum tensor of tachyon matter in the right-hand side of the equation (3) is reduced to a diagonal form $T_{\nu}^{\mu}=\operatorname{diag}(-\varepsilon, p, p, p)$, where the energy density $\varepsilon$ is positive

$$
\varepsilon=\frac{V(T)}{\sqrt{1-\dot{T}^{2}}}
$$

and the pressure $p$ is negative or zero

$$
p=-V(T) \sqrt{1-\dot{T}^{2}}
$$

Equation for the evolution of the scale factor follows from (3)

$$
\frac{\dot{a}^{2}}{a^{2}}=\frac{8 \pi G}{3} \frac{V(T)}{\sqrt{1-\dot{T}^{2}}} .
$$

Equation for the time-dependent rolling tachyon in an expanding universe follows from (田)

$$
\frac{\ddot{T}}{1-\dot{T}^{2}}+3 \frac{\dot{a}}{a} \dot{T}+\frac{V_{, T}}{V}=0 \text {. }
$$

Note that the tachyon potential enters the field equation in a combination $(\ln V)_{, T}$.

In the following sections we consider background solutions of equations (8) and (9) for two models of the tachyon potentials $V(T)$ from Figure 11. 


\section{FLUCTUATIONS IN ROLLING TACHYON}

The issue of stability of small scalar fluctuations is often essential in scalar field cosmology. In this section, we provide a formalism for treating linear inhomogeneous fluctuations in the tachyon field. Let us consider small inhomogeneous perturbation of the tachyon field $\delta T(t, \vec{x})$ around time-dependent background solution $T(t)$ of equation (9)

$$
T(t, \vec{x})=T(t)+\delta T(t, \vec{x}) .
$$

As we will see, for one of our examples of tachyon potentials $V(T)$, instability of tachyon fluctuations grows and becomes non-linear very quickly. Therefore first we write down the equation for fluctuations $\delta T(t, \vec{x})$ ignoring expansion of the universe and ignoring coupling of tachyon fluctuations with the metric fluctuations.

Linearizing the field equation (4) (without Hubble friction term) with respect to small fluctuations $\delta T$ and performing Fourier decomposition $\delta T(t, \vec{x})=\int d^{3} k T_{k}(t) e^{i \vec{k} \vec{x}}$ of the linear fluctuations, we obtain evolution equation for the time-dependent Fourier amplitudes $T_{k}(t)$

$$
\frac{\ddot{T}_{k}}{1-\dot{T}^{2}}+\frac{2 \dot{T} \ddot{T}}{\left(1-\dot{T}^{2}\right)^{2}} \dot{T}_{k}+\left[k^{2}+(\log V)_{, T T}\right] T_{k}=0
$$

Next we consider tachyon fluctuations coupled with metric perturbations in an expanding universe. Small scalar metric perturbations around expanding isotropic cosmology can be written in terms of Newtonian gravitational potential $\Phi(t, \vec{x})$ as

$$
d s^{2}=(1+2 \Phi) d t^{2}-(1-2 \Phi) a^{2}(t) d \vec{x}^{2} .
$$

Now we have to linearize the Einstein equations (3) and the field equation (4) with respect to small fluctuations $\delta T$ and $\Phi$.

Fortunately, the formalism for cosmological scalar fluctuations for the class of models which includes the theory (2) was developed in Ref. [5] (in connection with " $k$-inflation"). This is exactly what we need to pursue the investigation of small cosmological fluctuations with tachyon matter. Using results of [5], from (3) and (困) we obtain two coupled equations for the time-dependent Fourier amplitudes $T_{k}(t)$ and $\Phi_{k}(t)$,

$$
\left(\frac{T_{k}}{\dot{T}}\right)=\left(1-\frac{1}{4 \pi G} \frac{k^{2}}{a^{2}} \frac{\left(1-\dot{T}^{2}\right)^{3 / 2}}{V \dot{T}^{2}}\right) \Phi_{k},
$$

and

$$
\frac{\left(a \Phi_{k}\right)}{a}=4 \pi G \frac{V \dot{T}^{2}}{\left(1-\dot{T}^{2}\right)^{1 / 2}} \frac{T_{k}}{\dot{T}} .
$$

Introducing Mukhanov's variable $v_{k}$, which is related to the potential $\Phi_{k}$ as

$$
\frac{v_{k}}{z}=\frac{5 \varepsilon+3 p}{3(\varepsilon+p)} \Phi_{k}+\frac{2}{3} \frac{\varepsilon}{\varepsilon+p} \frac{\dot{\Phi_{k}}}{H}
$$

where energy density $\varepsilon$ and pressure $p$ are given by equations (6) and (7), $H=\frac{\dot{a}}{a}$, and 


$$
z=\frac{\sqrt{3} a \dot{T}}{\left(1-\dot{T}^{2}\right)^{1 / 2}},
$$

equations (13) and (14) can be reduced to a single second order equation for $v_{k}$

$$
v_{k}^{\prime \prime}+\left(\left(1-\dot{T}^{2}\right) k^{2}-\frac{z^{\prime \prime}}{z}\right) v_{k}=0,
$$

where prime $\left({ }^{\prime}\right)$ stands for derivative with respect to the conformal time $d \eta=d t / a(t)$. We will use this equation for analysis of coupled tachyon and metric fluctuations in an expanding universe for the tachyon potential $V(T) \propto e^{-T / T_{0}}$.

\section{NEGATIVE-PRESSURE TACHYON MATTER}

In this section, we consider the model with the potential $V(T)$ with its ground state at the finite value $T_{0}$, as it is sketched in the left panel of Figure 11. Let us assume that tachyon is rolling towards the minimum of the potential $T_{0}$. We will approximate the shape of the tachyon potential around the minimum by a quadratic form $V(T) \approx \frac{1}{2} m^{2}\left(T-T_{0}\right)^{2}$. Despite quadratic form of the potential, tachyon motion around $T_{0}$ is not harmonic, since $\ln V$ but not $V$ is involved in the tachyon equation of motion. For the same reason parameter $m$ drops out of the field equation (9). It is convenient to use tachyon field in units of $T_{0}$ and time $t$ also in units of $T_{0}$. Parameter $m$, however, is involved in the energy density of tachyon $\varepsilon \propto \frac{m^{2}}{t^{2}}$. The choice of $m \sim l_{s}^{-1} \sim M_{p}$ may bring the value of $\varepsilon$ to the required density of dark energy.

Numerical solution of the equations (9) and (8) reveals that tachyon very quickly, within time interval of several $T_{0}$, begins to oscillate around the minimum of the potential, as shown in the left panel of Figure 2. The amplitude of the oscillations is decreasing with time due to the Hubble friction term in the equation (9). The envelope curve (dashed line) in the left panel of Figure (2) shows the amplitude decreasing as $1 / t$. As we will see below, this time-dependence of the amplitude exactly corresponds to the (time-averaged) equation of state $\varepsilon / p$ which will be found for the tachyon matter in this model. Also, note that the period of oscillations is decreasing with time. Tachyon oscillations in this model are not only non-harmonic, but also non-periodic.

The instant value of the ratio of energy density (6) and pressure (17), $\frac{p}{\varepsilon}=\dot{T}^{2}-1$, is oscillating with time, as shown in the right panel of Figure 2. Although the amplitude of oscillations $T$ is decreasing with time, the amplitude of $\dot{T}$ is not changing with time as it is clear from the Figure 2 .

The period of oscillations is very small $\left(\sim T_{0}\right)$, so that only the average equation of state is important for cosmological evolution. To find it, we average $\dot{T}^{2}-1$ over several consecutive oscillations. The average value of $\frac{p}{\varepsilon}$, shown as the horizontal line at the right panel of Figure 2, is independent of time and equal to

$$
\left\langle\frac{p}{\varepsilon}\right\rangle=-\frac{1}{3} .
$$

For this type of the equation of state, average value of the energy density dilutes as $\langle\varepsilon\rangle \propto$

$a^{-2}$ with the expansion of the universe. The amplitude of the tachyon oscillations is then 


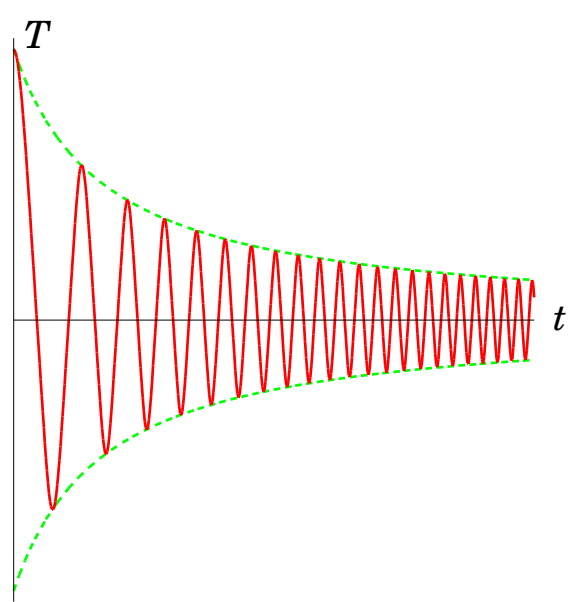

(a)

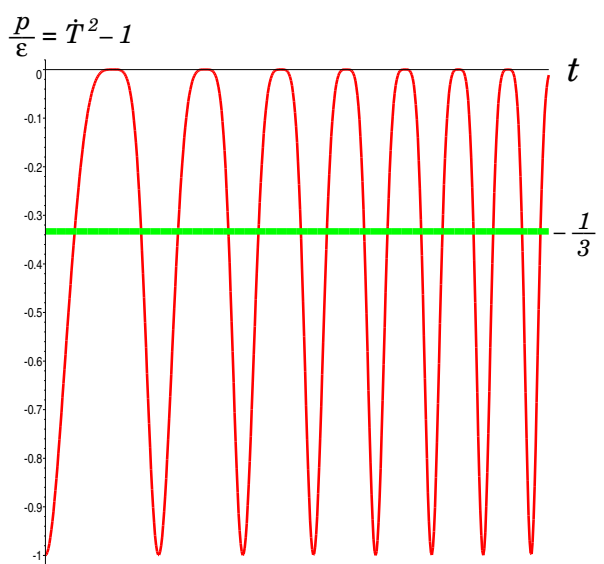

(b)

FIG. 2. (a) Background tachyon oscillations in the model with $V(T)=\frac{1}{2} m^{2}\left(T-T_{0}\right)^{2}$. (b) Background oscillations of tachyon equation of state. Constant horizontal line $\frac{p}{\varepsilon}=-\frac{1}{3}$ is the time-averaged equation of state.

decreasing as $1 / t$, which is compatible with numerical results. From (8) we find that the scale factor on average is $a(t) \propto t$. Note that equation of state similar to (18) takes place for a network of cosmic strings.

Equation of state (18) for the quadratic tachyon potential can be easily derived analytically. Indeed, assuming that tachyon is oscillating much faster than the universe expands, we can treat energy density $\varepsilon$ as adiabatic invariant, and write $\dot{T}^{2}=1-\frac{V^{2}(T)}{\varepsilon^{2}}$, where $\varepsilon$ is constant over several consecutive oscillations. Then the average value of $\dot{T}^{2}$ for quadratic potential is

$$
\left\langle\dot{T}^{2}\right\rangle=\frac{\int \dot{T}^{2} d t}{\int d t}=\frac{\oint\left(1-V^{2}(T) / \varepsilon^{2}\right)^{1 / 2} d T}{\oint\left(1-V^{2}(T) / \varepsilon^{2}\right)^{-1 / 2} d T}=\frac{2}{3},
$$

and so $\left\langle\frac{p}{\varepsilon}\right\rangle=\left\langle\dot{T}^{2}\right\rangle-1=-\frac{1}{3}$. If shape of the potential around the minimum is not quadratic, but a power-law $V \propto\left(T-T_{0}\right)^{n}$, the average equation of state is $\left\langle\frac{p}{\varepsilon}\right\rangle=-\frac{1}{n+1}$.

Although tachyon matter in the model has negative pressure, apparently it is short of explaining present acceleration of the universe. Combination of cosmological observations of CMB fluctuations, large scale structure clustering and high redshift supernovae constrains the equation of state to be lower than $\left\langle\frac{p}{\varepsilon}\right\rangle<-0.6$ [15].

As we will see in the next section, background tachyon dynamics in this model is unstable under small spatial fluctuations, and homogeneous tachyon oscillations will decay. It will be interesting to find what will be the final configuration of tachyon matter in this model and what may be its potential application to cosmology. 


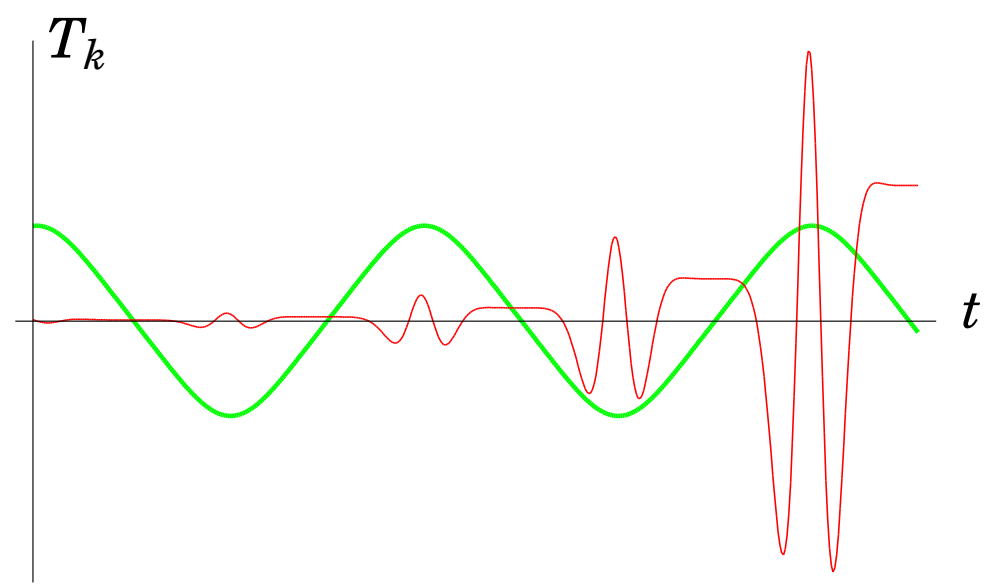

FIG. 3. Instability of fluctuations $T_{k}(t)$ in the model with quadratic potential (scales are linear).

\section{FLUCTUATIONS IN TACHYON MATTER WITH NEGATIVE PRESSURE}

It is expected that in realistic cosmological scenario tachyon field has small, quantum or classical, inhomogeneous fluctuations. In this section, we check the stability of tachyon fluctuations around the background solution discussed in the previous section. For the moment, let us ignore the expansion of the universe. Then we only have to solve equation (11) to find behaviour of fluctuations. Although formally some of the coefficients in equation (11) are singular when the background field $T(t)$ crosses zero in the case of quadratic potential, it is possible to switch to regular variables and overcome this technical inconvenience. Numerical solution of the fluctuation equation (for example for $k=10$ in units of $T_{0}$ ) is shown in Figure 3 .

General theory of linear equations with periodic coefficients predicts the presence of stability and instability bands of momenta $k$. For unstable modes, the amplitude is increasing exponentially as $T_{k}(t) \sim e^{\mu_{k} t}$. For the value of $k$ in Figure 3 the amplitude of fluctuations is increasing with time exponentially fast, by an order of magnitude in one background oscillation, say $T_{k}(t)$ increases by a factor of $10^{10}$ in ten oscillations! The physical reason is amplification due to the parametric resonance. This can be clearly seen if one rewrites equation (11) in the form of the oscillator-like equation, where the effective frequency is oscillating with time. This effect can be described by the theory of broad parametric resonance [14.

Since period of oscillation $\left(\sim T_{0}\right)$ is tiny compared to the cosmological time, and fluctuations become significant within several background oscillations, one can ignore expansion of the universe in this analysis. Thus we conclude that in the model with quadratic potential small tachyon fluctuations are exponentially unstable and background tachyon condensate decays into inhomogeneous configuration.

Decay of background tachyon condensate into inhomogeneous fluctuations does not necessarily mean that the universe becomes inhomogeneous. The tachyon fluid remains homogeneous as a whole, but not as the coherent condensate. Therefore it remains to be seen, based on the fully nonlinear analysis, what will be equation of state of the non-condensate tachyon fluid. 


\section{PRESSURELESS TACHYON MATTER}

In this section, we consider the model with the potential $V(T)=V_{0} e^{-T / T_{0}}$, with its ground state at infinity, as sketched in the right panel of Figure 1. Again, we can use dimensionless tachyon $T$ in units of $T_{0}$ and dimensionless time $t$ in the same units $T_{0}$. Background cosmological solutions of the equations (9) and (8) for this case very quickly (several $T_{0}$ ) enters the regime where tachyon is rolling very fast, and $\dot{T}$ approaches unity exponentially quickly

$$
T(t)=t+\frac{1}{4} a^{6} e^{-2 t}, \quad \dot{T}^{2}=1-a^{6} e^{-2 t},
$$

where $a(t)$ is the scale factor. The formula (20) applies, in particular, for radiation dominated stage where $a(t)=\sqrt{t}$ and tachyon does not contribute to gravity, and for $a(t)=t^{2 / 3}$ where tachyon gravitationally dominates. Without expansion of the universe, solution (20) corresponds to that of Sen [1].

In the above regime, tachyon potential is decreasing exponentially fast $V(T) \sim e^{-t / T_{0}}$. Remarkably, as it was discovered by Sen [1, 4, the energy density of tachyon matter is non-zero, while its pressure vanishes. In an expanding universe from (6), (7), (20) we have

$$
\varepsilon=\frac{V_{0} e^{-t / T_{0}}}{a^{3} e^{-t / T_{0}}} \rightarrow \frac{V_{0}}{a^{3}}
$$

while pressure vanishes

$$
p=-V_{0} e^{-t / T_{0}} a^{3} e^{-t / T_{0}} \sim-a^{3} e^{-2 t / T_{0}} \rightarrow 0
$$

In dimensional units one can estimate $\varepsilon \sim M_{p}^{2} / t^{2}$. In other words, rolling tachyon matter in an expanding universe is pressureless, its energy density dilutes as $\varepsilon \propto a^{-3}$, and its density may be tuned to the level of dark matter density of the universe. This makes tachyon matter with exponential potential a cosmological dark matter candidate.

\section{COSMOLOGICAL FLUCTUATIONS FOR PRESSURELESS TACHYON}

The crucial property of the cosmological dark matter without pressure is the growth of cosmological fluctuations, which form a developed large scale structure. The large scale structure of the universe is ranging from non-linear clustered halos of galaxies and clusters of galaxies, quasi-linear structures at scales of supercluster and voids, and linear fluctuations at very large scales. It is essential that at quasi-linear and non-linear stages dark matter is displaced from the homogeneous distribution due to the flows generated by the fluctuations of gravitational potential, and gravitationally bound halos have high velocity dispersions.

In this section, we investigate the clustering properties of the pressureless tachyon matter. We assume rolling tachyon matter domination and expansion rate of the universe is $a(t) \propto$ $t^{2 / 3}$.

We begin with linear analysis of cosmological fluctuations, using formalism of Section IIII. For a moment, consider the case without expansion of the universe and without coupling 
to gravitational perturbations. From equation (11) for exponential potential it follows that the fluctuations are not growing, $T_{k}=$ const.

Now let us consider tachyon fluctuations including expansion of the universe and coupling to gravitational potential $\Phi$. Substituting the background solution (20) for the pressureless tachyon matter into equations (17), one can see that the coefficient in the front of $k^{2}$ (which plays the role of the sound speed for the tachyon matter) vanishes exponentially fast. This means that the growth of linear tachyon fluctuations is scale free, similar to that of the standard cold dark matter scenario. Then the solution of (17) is $v_{k}=z$, and the lefthand side of equation (15) is constant. From this we immediately get the time evolution of fluctuations $\Phi_{k}$ and $T_{k}$

$$
\Phi_{k}(t)=\mathrm{const}, \quad T_{k}(t)=\Phi_{k} \cdot t
$$

Linear metric fluctuations are constant, similar to that in the cold dark matter scenario. However, the fluctuations in the tachyon field are growing, $\dot{T}_{k}=1$, in contrast to the simplified analysis above, where we neglected coupling to the metric fluctuations and expansion of the universe. The growth of tachyon fluctuations $T_{k} \propto t$ cannot be obtained without these ingredients. Thus, tachyon fluctuations are unstable due to the effects of gravitational instability in an expanding universe.

However, the linear approximation for rolling tachyon/gravity system works only during very short time interval (of order of tens of $T_{0}$ ). Indeed, let us inspect the energy density of tachyon matter in the model with exponential potential, not assuming it is homogeneous

$$
\varepsilon=\frac{V_{0} e^{-T / T_{0}}}{\sqrt{1-\dot{T}^{2}+\left(\nabla_{\vec{x}} T\right)^{2}}} .
$$

For fluctuations $\delta T$ we have $\delta T \simeq \Phi(\vec{x}) t$, where $\Phi(\vec{x})$ describes initial spatial profile of the fluctuations. The full tachyon field including fluctuations is $T(t, \vec{x})=\left(t+\frac{1}{4} a^{6} e^{-2 t}\right)+$ $\Phi(\vec{x}) t$. The numerator of expression (24) vanishes as $e^{-t}$, while the denominator evolves as $a^{6} e^{-2 t}-2 \Phi+\left(\nabla_{\vec{x}} \Phi\right)^{2} t^{2}$. The linear approximation works during very short time interval while $\left(\nabla_{\vec{x}} \Phi\right)^{2} \lesssim e^{-2 t}$. When this inequality breaks, linear analysis becomes insufficient. For cosmological fluctuations $\Phi \sim 10^{-5}$, linear theory is valid during time interval of order of $10 T_{0}$. Recall that for the standard particle dark matter scenario linear stage lasts during significant fraction of cosmological expansion.

One can try to use non-linear ansatz for the inhomogeneities of $T(t, \vec{x})$. Non-vanishing energy density (24) for rolling tachyon with numerator $V \sim e^{-t}$ is achievable if denominator is also vanishing as $e^{-t}$. Denominator $\sqrt{1-\dot{T}^{2}+\left(\nabla_{\vec{x}} T\right)^{2}}$ is not going to vanish unless we admit that $\left(\nabla_{\vec{x}} T\right)^{2}$ decreases faster than $\sim e^{-2 t}$. One of the working ansatz for inhomogeneous tachyon field is

$$
T(t, \vec{x})=t+f(\vec{x}, t) e^{-2 t},
$$

where $f(\vec{x}, t)$ weakly depends on $t$. This solution gives energy density (24) which is only spatially dependent. Solution for inhomogeneous tachyon was considered by Sen [16].

Now we will argue that fast rolling tachyon condensate with vanishing $p$ and nonzero $\varepsilon$ lacks nonvanishing gradients $\nabla_{\vec{x}} T$ which correspond to cosmic velocity, or displacement of 
tachyon matter. Indeed, energy-momentum tensor of tachyon condensate may be written in the hydrodynamic form

$$
T_{\mu \nu}=(\varepsilon+p) u_{\mu} u_{\nu}+p g_{\mu \nu},
$$

where corresponding fluid velocity is

$$
u_{\mu}=\frac{\nabla_{\mu} T}{\left(-\nabla_{\alpha} T \nabla^{\alpha} T\right)^{1 / 2}}
$$

energy density is given by (24) and pressure is $p=-V(T) \sqrt{1+\nabla_{\alpha} T \nabla^{\alpha} T}$.

Substituting here the solution (25), we find $u_{\vec{x}} \sim e^{-2 t}$. Thus, rolling tachyon condensate does not behave like a media of non-interacting particles. This is in contrast with the standard pressureless media of cold dark matter particles where large scale velocities and displacements of particles from their initial positions are growing with time.

\section{SUMMARY}

We considered cosmological solutions of rolling tachyon condensate $T$ for two models of tachyon potential $V(T)$. There are different levels at which one can theoreticize about $T(t)$ in expanding universe. Systematic approach suggests for us to begin with theory of tachyon field rolling down from the top of its potential. The curvature of the potential at the origin is negative, and we expect tachyonic instability of long wavelength fluctuations, similar to spinodal instability in usual field theory [11]. Thus there is an issue of initial conditions for rolling tachyon cosmology.

Suppose (by choice of initial conditions where $T$ is displaced from the origin) tachyon evolves towards its ground state as homogeneous condensate. We considered the model with minimum at finite $T_{0}$ and with quadratic approximation of $V(T)$ around the minimum. Then the background tachyon oscillates around the minimum with frequency of order of $1 / T_{0}$, and its (averaged over several oscillations) equation of state is $\frac{p}{\varepsilon}=-\frac{1}{3}$. However, we found from perturbation theory that tachyon fluctuations are exponentially unstable due to the tachyon self-interaction with background oscillations. It means that in this model homogeneous tachyon condensate decays. To answer the question what will be the resulting tachyon configuration, one has to go to the next level beyond the perturbation theory and to consider fully nonlinear problem of evolution of non-condensate tachyon fluid.

We also consider homogeneous tachyon rolling towards its ground state in the model with $V(T) \propto e^{-T / T_{0}}$, including expansion of the universe. In this model, the background tachyon condensate has vanishing pressure and finite energy density diluting as $\varepsilon \propto 1 / a^{3}$. Considering linear perturbations of tachyon field coupled with small metric perturbations, we found gravitational instability of tachyon field, $\delta T \propto t$. However, linear theory very soon (tens of $T_{0}$ ) becomes irrelevant. We argue that non-vanishing energy density of tachyon condensate is incompatible with large scale tachyon flows. Again, to make more certain conclusion about tachyon cosmology in this model, one has to use non-linear analysis to follow further evolution of inhomogeneities. 


\section{ACKNOWLEDGEMENTS}

We are grateful to D. Bond and A. Linde for useful comments. We thank NATO Linkage

Grant 975389 for support. A.F. was supported by NSERC, L.K. was supported by NSERC and CIAR. The work of A.S. in Russia was partially supported by RFBR, grants No 0202-16817 and 00-15-96699, and by the RAS Research Program "Astronomy". A.S. thanks CITA for hospitality during his visit.

[1] A. Sen, Tachyon matter, hep-th/0203265.

[2] G. W. Gibbons, Cosmological evolution of the rolling tachyon, hep-th/0204008.

[3] D. Kutasov, M. Marino and G. W. Moore, Some exact results on tachyon condensation in string field theory, JHEP 0010, 045 (2000); hep-th/0009148.

[4] A. Sen, Rolling tachyon, hep-th/0203211.

[5] C. Armendáriz-Picón, T. Damour and V. Mukhanov, k-inflation, Phys. Lett. B 458, 209 (1999); hep-th/9904075.

[6] J. Garriga and V. F. Mukhanov, Perturbations in k-inflation, Phys. Lett. B 458, 219 (1999); hep-th/9904176.

[7] A. Feinstein, Power-law inflation from the rolling tachyon, hep-th/0204140.

[8] A. Y. Kamenshchik, U. Moschella and V. Pasquier, An alternative to quintessence, Phys. Lett. B 511, 265 (2001); gr-qc/0103004.

[9] J. C. Fabris, S. V. B. Gonçalves, P. E. de Souza, Mass power spectrum in a universe dominated by the Chaplygin gas, astro-ph/0203441.

[10] L. Kofman, A. D. Linde and A. A. Starobinsky, Reheating after inflation, Phys. Rev. Lett. 73, 3195 (1994); hep-th/9405187.

[11] G. N. Felder, J. Garcia-Bellido, P. B. Greene, L. Kofman, A. D. Linde and I. Tkachev, Dynamics of symmetry breaking and tachyonic preheating, Phys. Rev. Lett. 87, 011601 (2001); hep-ph/0012142.

[12] P. B. Greene, L. Kofman and A. A. Starobinsky, Sine-Gordon parametric resonance, Nucl. Phys. B 543, 423 (1999); hep-ph/9808477.

[13] S. Mukohyama, Brane cosmology driven by the rolling tachyon, hep-th/0204084.

[14] L. Kofman, A. D. Linde and A. A. Starobinsky, Towards the theory of reheating after inflation, Phys. Rev. D 56, 3258 (1997); hep-ph/9704452.

[15] J. R. Bond, D. Pogosyan, S. Prunet, K. Sigurdson and the MaxiBoom collaboration, The Quintessential CMB, Past \& Future, in Proc. CAPP-2000 (AIP), CITA-2000-64; astro-ph/0011379.

[16] A. Sen, Field theory of tachyon matter, hep-th/0204143. 